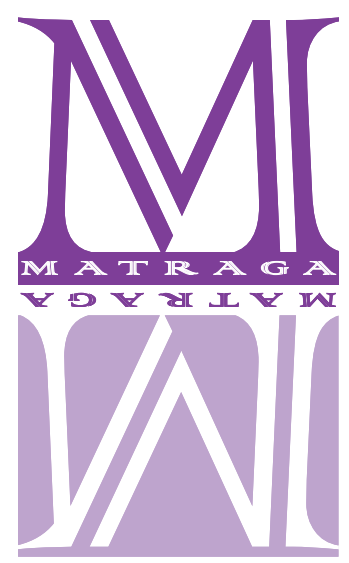

\title{
Beautés balnéaires : figuration et transfiguration du réel dans la représentation visuelle et textuelle à la fin du XIXe siècle
}

\author{
Susan Harrow \\ University of Bristol, Reino Unido \\ http://orcid.org/0000-0001-5879-1720
}

\section{RÉSUMÉ}

Des figurations de la beauté féminine - complexes et souvent contradictoires - réalisées par des artistes et des auteurs de la fin du XIXe siècle nous invitent à décrire trois courants de représentation visuelle et littéraire, tous tributaires de la querelle réaliste : primo, la beauté normative, idéalisée ; secundo, la beauté aggravée définie par la laideur et le grotesque ; tertio, la beauté quotidienne saisie dans son cadre " réel ", dans son vécu. Dans une perspective interdisciplinaire et transversale, j'interroge ces trois courants de la beauté figurative afin de cerner la modernité représentationnelle dans ses coïncidences et ses convergences inattendues, voire dans ses paradoxes. La beauté féminine est ici focalisée à travers des représentations de baignade et de bains dans une exploration qui comprendra des sites balnéaires (mer, océan, thermes, piscine, salle de bains), des supports tels des coquilles, des cuvettes, des baignoires, des tubs, et des accessoires de toilette féminine. Sortant des dichotomies traditionnelles érigées à partir d'esthétiques fortement contrastées, cette lecture croisée de textes et d'images vise à complexifier nos perspectives critiques en faisant dialoguer entre elles visions institutionnalisées, visions iconoclastes, et visions « quotidiennes ».

MOTS-CLÉS: Modernité; Beauté; Récit; Peinture académique; Le quotidien.

\section{Beauty bathing: figuration and transfiguration of the real in later nineteenth-century visual and textual culture}

\section{ABSTRACT}

The complex and often contradictory figurations of female beauty produced by artists and authors of the later nineteenth century suggest three strands of visual and literary representation, each variously related to the quarrel over realism: first, normative, idealized beauty; secondly, "aggravated" beauty and the aesthetic of laideur and the grotesque; and, thirdly, everyday beauty envisioned in the "real" world. Taking an interdisciplinary and transversal perspective to word and image, I examine these three strands alert to the coincidences, unanticipated convergences, and paradoxes of modern visual and textual representation. The literary and pictorial treatment of female beauty is angled here through representations of bathing in specific sites (sea, ocean, thermal baths, bathroom) and through material supports (shells, opulent baths, modest domestic tubs) and accessory objects. In a move away from traditional dichotomies and discriminations rooted in categorial perceptions of highly distinctive aesthetic styles, this comparative reading of texts and images seeks to complexify critical perspectives by bringing institutionalised visions, iconoclastic visions, and "everyday" visions into a fresh, productive dialogue with each other.

KEYWORDS: Modernity; Beauty; Narrative; Academic painting; The everyday. 


\section{Belezas balneares: figuração e transfiguração do real na representação visual e textual no fim do século XIX}

\section{RESUMO}

As figurações da beleza feminina - complexas e muitas vezes contraditórias - realizadas por artistas e autores do fim do século XIX nos convidam a descrever três correntes de representação visual e literária, todas elas relacionadas à querela realista: em primeiro lugar, a beleza normativa, idealizada; em segundo, a beleza agravada, definida pela feiura e pelo grotesco; em terceiro, a beleza cotidiana capturada em seu cenário "real", em sua experiência vivida. Numa perspectiva interdisciplinar e transversal, questiono essas três correntes de beleza figurativa, a fim de identificar a modernidade da representação em suas coincidências e convergências inesperadas, e mesmo em seus paradoxos. A beleza feminina é vista aqui através de representações de banhos em uma exploração que incluirá locais de banho (mar, oceano, termas, piscina, banheiro), suportes como conchas, bacias, banheiras, tinas e acessórios de toalete feminina. Rompendo com as dicotomias tradicionais erguidas com base em estéticas fortemente contrastantes, esta leitura cruzada de textos e imagens visa complexificar nossas perspectivas críticas, fazendo com que visões institucionalizadas, visões iconoclásticas e visões "do cotidiano" dialoguem.

PALAVRAS-CHAVE: Modernidade; Beleza; Narrativa; Pintura acadêmica; O cotidiano.

\section{Introduction}

Dans la tradition esthétique française, la représentation visuelle et littéraire de la femme nue (fin du XIX ${ }^{e}$ siècle) se décline, semble-t-il, selon des catégories résolument antithétiques. Primo, il y a la beauté idéale produite, promue, et promulguée par des peintres académistes et traitée par des poètes traditionnels et modernes (témoin la figuration resplendissante de la déesse hindoue Sita, évoquée dans "Lété » de Charles Cros : "Sous la gaze, d’or rayée, / Où son beau corps s'enveloppe / En sétirant, l’ennuyée / Ouvre ses yeux d’antilope » (vers 25-28, CROS, 2011, p. 62-63) ; secundo, se présente la beauté scandaleuse, produit de la transfiguration iconoclaste des avant-gardes refusant les poncifs de la beauté normative (nous songeons aux innovations audacieuses de Baudelaire dans «Une charogne " de Les fleurs du mal (T. 1, 1975, p. 29-31), de Rimbaud dans «Vénus anadyomène » de Poésies (1972, p. 22) ; et de Zola, qui évoque la dissolution de la chair abjecte de la courtisane éponyme dans la dernière scène de Nana (ZOLA, T. II, 1961) ; tertio, il y la beauté « quotidienne », valorisée par l'innovation naturaliste et impressionniste dans sa poursuite de sujets " réels ", authentiques, telles les blanchisseuses d'un Degas saisies dans la pratique d'un métier abrutissant. ${ }^{1}$ Au cours de cet article je viserai à interroger ces trois versants de la beauté balnéaire dans le but de cerner la représentation visuelle et littéraire dans ses coïncidences et ses convergences inattendues, alerte à la complexité et aux contradictions des réalités picturales et plus largement esthétiques.

\footnotetext{
${ }^{1}$ Le portrait de Gervaise Macquart dans L'Assommoir (1877) de Zola livre un exemple de la beauté synonyme d'honnêteté et de simplicité, et caractéristique des années d'avant la déchéance morale et physique de la blanchisseuse. Dans les années 1880 , Degas réalise une série de tableaux et de dessins autour du travail et de la corporéité - énergique et avachie - des repasseuses. Tableau accessible via le lien : <https://www.musee-orsay.fr/fr/oeuvres/repasseuses-1142>. Consulté le : 21 avr. 2021.
} 


\section{Physiologie et régime scopique sublimes}

Lart académiste français des années 1860-1880 se fonde sur la double esthétique du réel et de la sublimation du réel. J'entends "sublimation » ici dans les deux sens du terme : d'une part, il s'agit de rehausser, de perfectionner l'authentique forme féminine (ceci tirant sur l'idée sous-jacente d'une perfectibilité essentielle ou immanente) ; d'autre part, il s'agit de susciter des pulsions érotiques afin de les transformer en valeurs morale et esthétique en cultivant des préceptes de goût culturel normatif et d'une idée convenue du Beau et du Vrai. Les "Vénus » de facture académiste, figures créées et inlassablement reproduites par Alexandre Cabanel (1823-1889) et William-Adolphe Bouguereau (1825-1905) pour des consommateurs avides de fantaisies féminines pulpeuses déroulant dans des décors mythologiques et classicisants, font état d'un important travail de recyclage et de citation : l'art de Botticelli (La Naissance de Vénus, 1483-1485) est la référence privilégiée pour maintes Vénus et ondines issues du courant académiste, dont La Naissance de Vénus (1863) de Cabanel. ${ }^{2}$ Si l'esthétique d'un Cabanel ou d'un Bouguereau semble d'emblée refuser le réel contemporain, c'est toutefois la contemporanéité du réalisme pictural et du progrès technique - la photographie en particulier - qui sous-tend cet art aux décors intemporels où se déploient des visions "mythiques » focalisées sur des corps féminins offerts à la fétichisation d'acheteurs bourgeois et institutionnels férus d'un idéal suranné de la beauté, du goût, et du « raffinement » culturel, et non moins férus de la valeur commerciale de ce genre artistique tant prisé par des musées et des hauts patrons de l'art. ${ }^{3}$

Une dimension paradoxale de l'esthétique académiste relève ainsi de la conjonction de la mythologie « lointaine » à valeur allégorique, et d'un réalisme proximal qui décline la peau, ses qualités visuelle et haptique, sa pâleur, ses courbes et contours, dans la représentation d'un corps lisse, souple, accommodant, et qui met en valeur le visage dans son expressivité immédiate et " accessible » (on remarque le clin d'œil aguichant, typique des Vénus académistes posées entre pudeur mythique délicatement voilée et possibilité érotique perçue dans la frontalité du corps présentée au spectateur) : témoin La Naissance de Vénus (1879) de Bouguereau, tableau à la figuration irrésolue qui semble consacrer simultanément une déesse callipyge et une vedette de la nudité. ${ }^{4}$ Or, cette conjonction de l'éloignement et de la proximité fait découvrir la tension entre abstraction (abstraction du social, abstraction des moyens de l'art, valorisation du « raffinement » et de l'antique) et le jeu du contact direct avec le spectateur (fondé sur l'aspect fortement illusionniste, presque photographique de ces images), au point où le regardant aurait

\footnotetext{
${ }^{2}$ Tableau accessible via le lien suivant : <https://artsandculture.google.com/asset/the-birth-of-venus/awEIYqoUcAJ6TA?hl=en-GB>. Consulté le : 14 avr. 2021.

${ }^{3}$ Heather Dawkins (2002) analyse le corps féminin dans des contextes artistique, littéraire, commercial, et juridique, ouvrant une importante discussion sur la représentation non-normative du nu, forme subvertie par des journalistes-femmes et dé-fétichisée par des peintres telles Berthe Morisot et Mary Cassatt. Peter Brooks consacre un chapitre de sa monographie aux complexités du nu dans la culture du XIXe siècle, allant des modèles académistes perfectionnés jusqu'aux Vénus " kitsch 》 subversives créées par des auteurs et artistes modernes tels Zola et Manet (1993, p. 125). Voir l'important ouvrage de Richard Bretell sur les rapports entre l'art français et le commerce, le colonialisme, et la pornographie (1999). Pour des échos du style Cabanel ou Bouguereau dans la photographie érotique du tournant du XXe siècle, voir Alexandre Dupouy (2003).

4 Tableau accessible via le lien suivant : <https://www.musee-orsay.fr/fr/oeuvres/naissance-de-venus-16649>. Consulté le : 14 avr. 2021.
} 
l'impression de pouvoir presque toucher les surfaces de la peau peinte ; est mobilisée en même temps notre appréhension d'un corps qui rappelle fortement la statue de cire et, par conséquent, la céroplastie et donc l'anatomie, puisque ces Bacchantes, Vénus et Psyché assorties ne cessent de nous renvoyer à l'art " naturaliste " inspiré par des amphithéâtres d'écoles de médecine où la forme de prédilection est la patiente nue, souvent la femme hystérique, objet du regard et du toucher des médecins renommés et de leur entourage : songeons au tableau d'Henri Gervex, Le Docteur Péan, avant l'opération (1887). ${ }^{5}$ Je reviendrai plus loin à l'anatomie par rapport au mode iconoclaste. ${ }^{6}$

Un phénomène pictural parallèle au courant mythologisant et classicisant d'un Bouguereau ou d'un Cabanel s'étend outre-Manche dans l'art victorien et post-victorien des années 1880 jusqu'aux années 1910. Prenons l'art du peintre académicien Lawrence Alma-Tadema (18361912) : Vénus et ondines sont ici transposées du cadre explicitement mythique à des intérieurs luxueux inspirés de sites et monuments romains antiques. A Favourite Custom (1909) nous fait découvrir des espaces marmoréens, hermétiquement clos (ou presque), situés de façon ambiguë entre l'antiquité à laquelle ils font référence et la modernité évocatrice d'un bienêtre aux allures " nobles »..$^{7}$ Ces lieux historiques " réels »- sanctuaires de la sensualité (et de lélitisme social) - sont propices à l'évasion et à la rêverie connotées par la souplesse des corps féminins diaphanes et par une gestuelle à la fois vivace et reposée. ${ }^{8}$ Il y a, en parallèle, un fort déploiement de la notion implicite et explicite du «cadre » (cadres pictural, sculptural, architectural ; marbres avec leurs valeurs d’ordre, d'harmonie, de pérennité), indices de la survalorisation de cultures classiques recyclées. Le peintre anglais transforme la coquille de Vénus, support de la déesse depuis Botticelli, en un espace raréfié, immémorial, et pourtant quotidien, en citant la référence classique et archéologique aux pratiques culturelles du frigidarium. C'est l'antiquité romaine (celle des thermes de Stabies de Pompéi) qui est mise en valeur dans cet espace à la fois an-historicisé et profondément historique, comme Elizabeth Prettejohn l'a démontré au cours d'une lecture transculturelle de la représentation de Rome, capitale vue et revue par un Alma-Tadema alerte au paradoxal, alerte surtout à la modernité du monde antique, comme à l'ancienneté du moderne : ce traitement double aurait son équivalent dans le Paris du Second Empire analysé par des commentateurs tels Baudelaire et Maxime Du Camp, tous deux attentifs aux correspondances entre le moderne et l'ancien (PRETTEJOHN, 2002, p. 115-129). Le bassin du frigidarium au centre du tableau d'Alma-Tadema est le théâtre

\footnotetext{
5 Tableau accessible via le lien suivant : <https://www.musee-orsay.fr/fr/oeuvres/avant-loperation-9549?cHash=85ed4cb809\&tx_commentaire_pi $1 \% 5 B$ BidLi $\% 5 D=509 \& t x \_c o m m e n t a i r e \_p i 1 \% 5 B f r o m \% 5 D=841>$

$<$ https://www.musee-orsay.fr/fr/oeuvres/avant-loperation-9549?cHash=85ed4cb809\&tx_commentaire_pi1\%5BpidLi\%5D=509\&tx_commentaire_pi1\%5Bfrom\%5D=841>. Consulté le : 21 avr. 2021.

${ }^{6}$ Pour une étude historique et culturelle de cet art insolite au croisement de la médecine, de la technique, et de l'esthétique, voir Latour (2020).

${ }^{7}$ Le site internet du musée Tate (Londres) souligne la dette du peintre envers l'archéologie du XIXe siècle et les fouilles des thermes de Stabe de Pompéi (1824). Voir : <https://www.tate.org.uk/art/artworks/alma-tadema-a-favourite-custom-n02675>. Consulté le : 21 avr. 2021.

${ }^{8}$ Voir son tableau intitulé Un Poète favori (1888), dans Lady Lever Art Gallery, Liverpool. Tableau accessible via le lien suivant : $<$ https://www.alamyimages.fr/lawrence-alma-tadema-le-poete-favori-peinture-1888-image265953804.html>. Consulté le : 21 avr. 21.
} 
de la peau, et le jeu spontané des femmes dans l'eau semble libérer et moderniser les corps, effectuant un rapprochement du passé et du présent. Femmes et eau, il y a transparence et renforcement mutuel entre les modèles et le médium aquatique; et, surtout, il y a la qualité transparente de l'eau qui, à la fois, voile et dévoile son objet, à l'instar d'une esthétique picturale irrésolue, oscillant entre le clos et le clair, le limpide et l'ambigu, l'actif et l'indolent.'

Peau lisse, pierre lisse : la vision classique de la beauté marmoréenne est doublée par une vision de la chair, de la chaleur corporelle (rehaussée dans la fraîcheur du frigidarium), de l'incarnation (de baigneuses "antiques" transfigurées par la réalité charnelle des modèles d'époque qui posaient pour le peintre). En quelque sorte, le baptême de la beauté est fondé sur un idéal classique accommodé à lère de l'impérialisme triomphant - celui de l'antiquité romaine de la représentation imagée, et celui de lépoque (post-)victorienne de la production et de la première réception du tableau. Des bains de luxe - espace exclusivement féminin dans $A$ Favourite Custom - permettent la mise en valeur de la beauté sublime, rehaussée, et, pourtant, d’apparence « réelle». En outre, peindre ces espaces privilégiés permet la mise en scène d'une certaine notion de classe sociale. Intouchée par le dehors, c'est la beauté du sanctuaire, voire du sanctum (au sens latin, lieu du purifié) qui est valorisée, ce qui nécarte pas pour autant des soupçons d'érotisme et d'auto-érotisme. ${ }^{10}$

Le topos de la baignade au sanctuaire est repris vers la même époque par Émile Zola dans La Curée (1872) : dans le cabinet de toilette de Renée Saccard trône sa prestigieuse baignoire, objet décrit longuement, luxueusement, par le narrateur. Ici, cependant, l'évocation de l'opulence Second Empire où luxe frôle luxure (Renée a déjà cédé au désir adultère dans l'étreinte de son beau-fils Maxime) tourne vite à un discours discrètement critique : sur le mode mimétique, le récit naturaliste décline tous les éléments de la ferveur classicisante (et de l'art académiste mythologisant) d'un régime obsédé par le fastueux et le pompier, où la mise en scène de la « grande nudité » féminine, suprême artifice de l’empire de Napoléon III, est exposée ici comme un leurre ; c'est la fausse réalité dans laquelle Renée se laissera glisser :

[...] le cabinet avait un coin délicieux, et ce coin-là surtout le rendait célèbre. En face de la fenêtre, les pans de la tente s'ouvraient et découvraient, au fond d'une sorte d'alcôve longue et peu profonde, une baignoire, une vasque de marbre rose, enfoncée dans le plancher, et dont les bords cannelés comme ceux d'une grande coquille arrivaient au ras du tapis. On descendait dans la baignoire par des marches de marbre. Au-dessus des robinets d'argent, au col de cygne, une glace de Venise, découpée, sans cadre, avec des dessins dépolis dans le cristal, occupait le fond de l'alcôve. Chaque matin Renée prenait un bain de quelques minutes. Ce bain emplissait pour la journée le cabinet d'une

\footnotetext{
${ }_{9}^{9}$ C'est un style qui ne cesse de se perpétuer : témoin les marchandises proposées par des musées nationaux et internationaux (tapis de souris, parapluies, carte de vœux, chemises pour iPad) sous forme d'objets de consommation culturelle qui s'approprient l'image de la féminité séduisante.

10 Voir Rosemary Barrow (1997-98, p. 183-202). Dans une perspective révisionniste, Barrow explore les valeurs inter-artistiques d'une esthétique qui puise dans la poésie de Catulle et d'Horace pour créer des tableaux intrigants qui oscillent entre transparence et opacité du contenu sensuel, voire érotique. En outre, Barrow fait ressortir un écho intertextuel de Suétone (Vie de Néron) dans La Faute de l'abbé Mouret (1875) de Zola où Albine meurt intoxiquée par le parfum des roses ; la relation florale-olfactive dans l'évocation de la sensualité enivrante des scènes antiques est sublimée dans The roses of Heliogabalus (1888) d'Alma-Tadema.
} 
moiteur, d'une odeur de chair fraîche et mouillée. Parfois, un flacon débouché, un savon resté hors de sa boîte mettaient une pointe plus violente dans cette langueur un peu fade.

La jeune femme aimait à rester là, jusquà midi, presque nue. La tente ronde, elle aussi, était nue. Cette baignoire rose, ces tables et ces cuvettes roses, cette mousseline du plafond et des murs, sous laquelle on croyait voir couler un sang rose, prenaient des rondeurs de chair, des rondeurs dépaules et de seins ; et, selon l'heure de la journée, on eût dit la peau neigeuse d'un enfant ou la peau chaude d’une femme. C’était une grande nudité (ZOLA, T. 1, 1960, p. 479-480).

La valorisation de l'antiquité passe par le perfectionnement du modèle féminin à la peau sublimée par le pinceau de l'artiste, et ensuite par le toucher quasi-haptique du regard du spectateur. Les Vénus et nymphes lisses réalisées par Bouguereau et Cabanel partagent des qualités mimétiques, sensuelles, et inlassablement allégoriques ; elles sont positionnées entre la réalité et l'idéalisme auquel elles font allusion. Alma-Tadema, lui, attire l'œil du spectateur sur la peau de façon audacieuse dans The Tepidarium (1881) : la baigneuse tient, dans sa main droite, un strigile (motif caractéristique du bain romain où ce racloir sert à extirper de la peau les impuretés [sueur, poussière, huile préalablement appliquée]). ${ }^{11}$ La pratique quotidienne du travail sur la peau (le travail du sujet et de ses servantes faisant ici écho au travail du peintre sur la toile) est représentée à la fois par un mode d’observation "objectif » et une approche plus proximale, voire haptique : focalisée sur la beauté perfectionnée du modèle, cette image allie un certain réalisme documentaire à des valeurs esthétique et érotique (avec effleurement autoérotique implicite) dans une mise en scène du processus esthétique (dans la double acception du terme " esthétique "). ${ }^{12}$ Un parallèle inattendu se dessine ici entre peinture académiste et peinture moderne : témoin le célèbre tableau de Manet, Nana (1877), portrait de la courtisane qui se maquille, qui tient dans sa main les outils de sa toilette, et qui « tient » son client bourgeois, l’eil morne de ce personnage caricatural, radicalement coupé par le bord du tableau, fixant la demi-mondaine dans la performance des gestes quotidiens de sa toilette. ${ }^{13}$ Comme le modèle représenté au tepidarium d'Alma-Tadema, la courtisane de Manet présente les outils de sa construction (en l'occurrence, la houppe, le rouge à lèvres, la psyché) ; ce tableau, de facture combien différente de celui du peintre victorien anglais, explore la beauté dans sa réalité vécue, comme événement et comme la création d'une fiction, et en ceci les tableaux anglais victorien et français moderne se rejoignent. La «Nana » de Manet et la baigneuse du Tepidarium font un clin d'œil au regardant, signalant l'ironie perceptible entre la beauté idéale, d'une part, et sa production matérielle et sa qualité factice, d'autre part ; dans les deux cas, il s'agit d'un art conscient de ses propres méthodes et astuces, et qui signale le leurre qui lui est propre. Une lecture au second degré s'y dessine ainsi...

\footnotetext{
${ }_{11}$ Tableau accessible via le lien suivant : <https://www.vads.ac.uk/digital/collection/NIRP/id/30135/>. Consulté le : 21 avr. 2021.

${ }^{12}$ Selon la légende, la compagnie Pears, fabricant de savon anglais, envisageait d'employer cette image dans ses publicités, mais s'est ravisée en raison de l'érotisme et de l'auto-érotisme qui s'y laissent deviner.

${ }_{13}$ Tableau accessible via le lien suivant : <https://online-sammlung.hamburger-kunsthalle.de/en/search?term=Nana>. Consulté le : 21 avr. 2021.
} 
Dans le tableau d'Alma-Tadema, la plume d’autruche, déployée par la baigneuse, couvre délicatement son sexe, tout en l'exposant d'une manière suggestive et esthétique (geste féminin, voire féminisé), ce qui serait à comparer et à contraster avec la main d'Olympia (Manet, Olympia, 1863), image qui, sur un tout autre mode, attire l'attention sur le pubis caché de la courtisane et, ce faisant, déconstruit le regard du voyeur. ${ }^{14}$ Dans The Tepidarium, la peau dours serait le signe de l'élément sauvage maîtrisé, mais la contiguïté de la peau d'animal et de la peau de femme, leur rapprochement haptique, ne cesse d'articuler un récit de désirs inassouvis et volatils; la nature originelle, domptée par la culture et la bienséance (sociale, esthétique), menace de resurgir. ${ }^{15}$ Ainsi, dans des tableaux de facture radicalement différente - Manet iconoclaste, Alma-Tadema adapté au goût du jour bourgeois - font découvrir une réalité complexe, équivoque, qui ne cesse de troubler les classifications académiste et innovante. ${ }^{16}$

La beauté idéale est incorporée de façon souvent sceptique ou ironique par la modernité littéraire dans son projet d’approfondir et de mettre à nu des réalités multiples, complexes, souvent contradictoires : l'esthétique moderne intègre souvent une citation infléchie ou franchement transgressive de la beauté sublime. Prenons ici un exemple tiré de Madame Bovary (1857) de Flaubert. Dans sa poursuite des poncifs du romantisme, Emma Bovary suscite dans l'esprit de son amant Léon une analogie "parlante ", voire plusieurs. Dans la troisième partie du roman, au chapitre 5 , nous lisons, selon la double perspective de l'hérö̈ne et de son amant, tous deux à la recherche de possibilités supérieures confectionnées, la réalité intertextuelle, inter-artistique, citationnelle, qui constitue « Emma» :

[Emma] était l'amoureuse de tous les romans, l'héroïne de tous les drames, le vague elle de tous les volumes de vers. [Léon] retrouvait sur ses épaules la couleur ambrée de l'Odalisque au bain; elle avait le corsage long des châtelaines féodales; elle ressemblait aussi à la Femme pâle de Barcelone, mais elle était par-dessus tout, Ange ! (FLAUBERT, T. 3, 2013, p. 533).

Les épaules ambrées d'Emma semblent évoquer la coloration du modèle d'Ingres dans La Grande Odalisque (1814), ou la coloration de tel autre modèle de tel autre peintre, car le narrateur ne livre aucune précision sur le créateur de cette odalisque (im)mémorielle. ${ }^{17}$ Cette série d'analogies et de parallèles expose le contenu hybride et réducteur à partir duquel Léon construit sa fantaisie "Emma ", qui devient, par l'amalgame de ces références et allusions culturelles inter-artistiques, une création hybride, morcelée, postmoderne, ou peu sén faut. La «vraie » Emma, éclipsée par la liste des allusions tirées du bagage culturel éclectique de Léon,

\footnotetext{
14 Tableau accessible via le lien : <https://artsandculture.google.com/asset/olympia/ywFEl4rxgCS01Q?hl=en\%20l>. Consulté le : 21 avr. 2021.

15 Pour une étude des rapports entre art, colonialisme, relations humaines-animales, et goût culturel, voir Colley (2014).

${ }^{16}$ Faire la comparaison avec la beauté figurée par Manet : sa courtisane Olympia, inspirée du modèle de Vénus d'Urbin (1538) de Titien, se vante de son statut d'objet perfectionné (parce qu'orné : collerette, souliers, fleurs, nœud dans les cheveux), et se délecte à dessiner avec sa main un geste auto-érotique. Un écart s'instaure entre l'art des peintres victoriens aux valeurs " pures ", éthérées, et l'art moderne avec sa mise en valeur de la texture de son médium, mais ces esthétiques d'apparence fortement contrastée laissent découvrir une réciprocité inattendue sur le plan d'une beauté située, historicisée et composée, et qui s'appuie sur ses matériels et ses modes préparatifs.

17 La Grande odalisque est accessible via le lien suivant : <https://collections.louvre.fr/en/ark:/53355/cl010065566>. Consulté le : 21 avr. 2021. Voir la discussion de ce tableau dans Mirzoeff (1995).
} 
est récupérée par la mixité référentielle déclinée à tour de rôle par la valeur artistique exotique de lodalisque ; par le pouvoir de séduction de la courtisane ; par la vision surannée de la dame médiévale ; et par la valeur spirituelle, éthérée du superlatif terminal "par-dessus tout, Ange ».

\section{Physiologie et régime scopique aggravés : la beauté dé-idéalisée}

La beauté féminine est interrogée, métamorphosée, radicalement redéfinie dans des poèmes de Baudelaire tels "La charogne ", "Les petites vieilles ", et "Une mendiante rousse " de Les Fleurs du mal. Figurée paradoxalement, la beauté moderne incite à de nouvelles connaissances, à une conscience autre, lucide, sceptique, en quête d'une réalité plus profonde, plus « difficile ", souvent inédite; c'est la vérité de la vision d’avant-garde.

La mutabilité de la beauté est fruit de la mobilité des idées et mentalités prônée par Baudelaire, analyste et inventeur de réalités audacieuses, où les distinctions se dissolvent, où les anciennes certitudes et dichotomies disparaissent. La beauté stimule désormais le goût du paradoxe et de l'irrésolu. Dans « L'hymne à la beauté », le poète s'interroge en interrogeant la beauté :

Viens-tu du ciel profond ou sors-tu de l'abîme,

O Beauté ? ton regard, infernal et divin,

Verse confusément le bienfait et le crime,

Et lon peut pour cela te comparer au vin.

Que tu viennes du ciel ou de l'enfer, qu'importe,

Ô Beauté ! monstre énorme, effrayant, ingénu !

Si ton œil, ton souris, ton pied, mouvrent la porte

D’un Infini que j’aime et n’ai jamais connu?

De Satan ou de Dieu, qu'importe? Ange ou Sirène,

Qu'importe, si tu rends, - fée aux yeux de velours,

Rythme, parfum, lueur, ô mon unique reine ! -

L'univers moins hideux et les instants moins lourds?

(vers 1-4 ; 21-28, BAUDELAIRE, T. 1, 1975, p. 24-25)

En refusant les antithèses, refuge rhétorique du raisonnement rigide, en défaisant les anciennes valeurs, le poète cherche à libérer l'esprit des anciennes entraves de la pensée, et annonce une réalité complexe, mobile, qui sera le catalyseur de visions inédites et de vérités nouvelles susceptibles d'enrichir l'existence en approfondissant et en diversifiant la notion de réalité.

C'est sur le mode iconoclaste que Rimbaud, à son tour, s'en prendra à la vision utopique de la beauté, et plus généralement à la doxa esthétique, culturelle et sociale de son époque. Rimbaud cible le modèle académiste, que l’on pourrait dire « universaliste » (selon la perspective 
oculo-centrique, occidentale) - celui de la Vénus anadyomène, topos pratiqué, entre autres, par des peintres romantiques inspirés par l'art de la Renaissance, tels Théodore Chassériau (Vénus marine ou Vénus Anadyomène, 1838).

Dans « Vénus anadyomène " Rimbaud (1972, p. 22) pratique une modernité sulfureuse, exposant dans ce sonnet à scandale une corporéité féminine dé-idéalisée, aggravée, aggravante, et ainsi bafouant les valeurs culturelles bourgeoises. Muni de sa loupe analytique, le poète adopte la posture d'un clinicien dans son travail en gros plan sur un corps des plus décadents et déchus : le sonnet poursuit l'examen minutieux des plis de la chair squameuse aux proéminences osseuses de l'anti-Vénus, sujet qui dénonce le régime scopique en en mimant les modes et procédés. Cette masse monstrueuse surgit d'une baignoire rouillée, comme d'un cercueil dégradé, comme du sarcophage de la beauté classique :

\section{Comme d'un cercueil vert en fer blanc, une tête \\ De femme à cheveux bruns fortement pommadés \\ D’une vieille baignoire émerge, lente et bête, \\ Avec des déficits assez mal ravaudés; \\ Puis le col gras et gris, les larges omoplates \\ Qui saillent; le dos court qui rentre et qui ressort; \\ Puis les rondeurs des reins semblent prendre l'essor ; \\ La graisse sous la peau parait en feuilles plates :}

L'échine est un peu rouge, et le tout sent un goût

Horrible étrangement ; on remarque surtout

Des singularités qu'il faut voir à la loupe...

Les reins portent deux mots gravés : CLARA VENUS;

- Et tout ce corps remue et tend sa large croupe

Belle hideusement d'un ulcère à l'anus

(vers 1-14, RIMBAUD, 1972, p. 22)

Le discours anatomique ( l'échine », " les larges omoplates ", "l'anus », « l'ulcère ») balaye d’un coup le langage privilégié de la beauté classique et le discours du bon goût. Au moyen du registre médical, les procédés des sciences expérimentales sont ici évoqués et détournés afin de précipiter la subversion des poncifs de la beauté. L'objet corporel est présenté dans une mise en scène de ce qui est - ou devrait être - gardé hors-scène (c'est l'ob-scène, au sens étymologique : l’impudeur, le déchet, la plaie prévalent dans la réalité outrée qu'est Vénus anadyomène). Le médium du poème matérialise ainsi, sous nos yeux, l'objet scandaleux. Le rêve de la beauté normative est bafoué par la lucidité critique moderniste (représentée par la loupe que manie le narrateur anatomiste de Vénus ici ré-figurée, - défigurée - à des fins parodiques) : la prétendue objectivité pathologique et ironique contrecarre la notion même de séduction visuelle et de plaisirs scopiques faciles. L'offensive est ainsi lancée par Rimbaud contre l'art académiste, contre la culture officielle, contre une certaine construction de la femme, et ainsi contre toutes ces 
Vénus normatives qui prolifèrent à l'époque, et depuis. L'inscription " CLARA VENUs ", gravée dans la peau grotesque, fait allusion aux idéaux marmoréens surannés, désormais tournées en dérision, ternies, maculées. L'inscription signe ironiquement la vision proférée par le poète moderne, inventeur de l'objet abject qui, repoussant et fascinant, nous détourne et nous interpelle simultanément.

La beauté rimbaldienne se définit comme "beauté sauvage " : beauté moderne, iconoclaste, osée, incomparable. C'est surtout une beauté paradoxale, oxymorique. Dans un autre médium, l'œuvre de Degas fait ressortir une similitude profonde avec la vision rimbaldienne contreculturelle. Les monotypes réalisés par Degas, représentant des scénarios de maison close des années 1870, mettent en scène des corps féminins qui suintent, qui dépassent les limites morales et esthétiques normatives dans leur lourdeur et leur excès : ces traits sont réalisés par le brouillage et la maculation de la ligne, signes d'un art qui refuse la clarté classique et répudie la netteté monologique, fondant de nouvelles visions, plus proches de la réalité dé-idéalisée.

\section{La corporéité « naturelle » : innovation impressionniste autour de réalités quotidiennes}

Concept présumé intermédiaire dans l'ordre des valeurs esthétiques, la beauté " naturelle » serait située entre la vision stéréotypée des tableaux de Bouguereau et de Cabanel et la transfiguration iconoclaste des modernes tels Rimbaud. Des représentations " naturelles » de corps de baigneuses et de nus, captés dans des cadres quotidiens par le courant réaliste et impressionniste de la fin du XIX ${ }^{e}$ siècle, ne seraient pas pour autant neutres. Cela dit, elles seraient peut-être moins misogynes que la critique d’art féministe des années 1980-1990 a voulu prétendre, surtout si l'on comprend l'impressionnisme comme, entre autres, une offensive lancée contre les valeurs périmées de la beauté idéale, plutôt qu'une attaque contre la femme (et les femmes en général). ${ }^{18}$

La représentation de femmes à leur toilette dans des tableaux de Degas (1834-1917), sujet abordé au premier degré, révèle la banalité - et la profonde vérité - du geste quotidien. Mais l'art moderne - ses techniques et perspectives, son audace - est loin dêtre banal. Prenons Le Tub (1886) : ce pastel propose une image fracturée et recomposée d'une femme accroupie avec, à son côté gauche, la tablette, surface plane qui instaure une nouvelle géométrie susceptible de déstabiliser les perspectives habituelles. ${ }^{19}$ S’agit-il de la femme visuellement prise à son insu, ou de la femme qui tourne le dos à son observateur, qui refuse son regard fétichisant ? Un certain hermétisme ressort de ce tableau : ses deux plans ou panneaux font jouxter sujet humain et nature morte ; le corps de la femme, d'une part, et des objets accessoires (peigne, cruche, bassin), d’autre part. Refusant implicitement les thèses d'un Degas « misogyne », Julia Kristeva

\footnotetext{
18 Kendall et Pollock (1992) visent à exposer la présumée misogynie de l'art de Degas en focalisant, de façon partielle et partiale, « le voyeurisme » et « la vue patriarcale », à l'exclusion d'une visée plus large. Plus récemment, des approches hybrides, plurivalentes, ont réussi à ouvrir des perspectives sensibles à la modernité et à l'humanité du peintre, dont Kathryn Brown (2017). ${ }^{19}$ Tableau accessible via le lien suivant : <http://www.kristeva.fr/degas.html>. Consulté le : 21 avr. 2021.
} 
a longuement commenté ce tableau, sensible à l'humanité du peintre et à son immersion dans le réel du geste quotidien de la toilette, et attentive à l'intime articulation de valeurs aussi haptiques que visuelles. Kristeva écrit :

Ce $N u$ na pas de visage, il nous tourne le dos et ne suppose pas de public. L'œil plongeant, le geste enveloppant du peintre lui confère la retenue de Rembrandt et la vibration de Véronèse, deux mấtres que cet impressionniste [Degas] a longuement étudiés, copiés, incorporés. Son pastel invite à une intimité resserrée sur elle-même, inaccessible parce que réelle.

Cadrée dans son tub, la nudité ne s’offre donc pas à la vue, mais semble sévider d’elle-même, en se repliant, accroupie sur le miroir dépoli d’une énorme poêle à frire en fonte qui ne la regarde pas. $\mathrm{Ni}$ séduction ni masturbation mais, à travers elles ou sans elles, Degas ausculte une intériorité invisible qui se dérobe, se fuit, se refait et se défait. Il touche la nôtre, dans laquelle il n’y a rien à voir, seulement à ressentir l'apparition-disparition qui frémit « à la racine de l'Être, à la source de l'impalpable sensation », aurait dit Cézanne. ${ }^{20}$

La juxtaposition des deux plans - la coïncidence de deux réalités qui dépendent l'une de l'autre et semblent par ailleurs s'exclure mutuellement - est la marque d'une modernité poussée. La coquille de l'antique Vénus est ici transformée en un banal « tub» et en une surface et un fond, explicitement non-nacrés, supports qui refusent d'articuler une image spéculaire d'appropriation facile : la quasi-opacité du fond de tub, doublée par la surface plane de létagère qui se projette vers l’oil du spectateur, semble bloquer le regard fétichisant ou, du moins, le faire dévier. Témoin l'intériorisation du regard de la femme sur elle-même ; absorbée par son geste habituel de se laver, se donnant à sa seule intention, elle s'abstrait de notre regard, de notre tentative de récupération. La focalisation de l'image sur ses propres moyens - comme sur son propre sujet - dénoue la « simple » représentation du réel pour faire un pas décisif vers un art plus abstrait, autotélique.

L'abstraction de l'art au sein de cette esthétique de la beauté naturelle, quotidienne peut sembler chose rare. Nous songeons à certains tableaux réalisés par Berthe Morisot (1841-1895), dont Femme à sa toilette (1875/80), où les soies habillant la figure féminine semblent fondre dans les tissus et les décors gris perle, rose et argent d'un espace pictural où les images spéculaire et réelle ne font qu'une. ${ }^{21}$ C'est une scène qui vaut d'être comparée avec celle où l'éponyme Nana (Zola, Nana) se délecte à sonder le flou et le fugitif de sa propre image spéculaire dont la "réalité » se dissout en abstractions multiples (jeune fille en fleurs, Vénus pulpeuse, almée égyptienne), voire en illisibilité :

Nana sétait absorbée dans son ravissement d'elle-même. Elle pliait le cou, regardant avec attention dans la glace un petit signe brun quelle avait au-dessus de la hanche droite; et elle le touchait du bout du doigt, elle le faisait saillir en se renversant davantage, le trouvant sans doute drôle et joli, à cette place. Puis, elle étudia d’autres parties de son corps, amusée, reprise de ses curiosités vicieuses

\footnotetext{
${ }^{20}$ L'article de Julia Kristeva, paru sur son site internet officiel, est accessible via le lien suivant : <http://www.kristeva.fr/degas.html>. Consulté le : 21 avr. 2021.

21 Tableau accessible via le lien suivant : <https://www.artic.edu/artworks/11723/woman-at-her-toilette>. Consulté le : 21 avr. 2021.
} 
d’enfant. Ça la surprenait toujours de se voir ; elle avait l'air étonné et séduit d'une jeune fille qui découvre sa puberté. Lentement, elle ouvrit les bras pour développer son torse de Vénus grasse, elle ploya la taille, s'examinant de dos et de face, s’arrêtant au profil de sa gorge, aux rondeurs fuyantes de ses cuisses. Et elle finit par se plaire au singulier jeu de se balancer, à droite, à gauche, les genoux écartés, la taille roulant sur les reins, avec le frémissement continu d'une almée dansant la danse du ventre (ZOLA, T. II, 1961, p. 1270).

L'impressionnisme de Morisot et le naturalisme de Zola semblent, dans les deux cas, mettre en scène la transition esthétique de la mimésis vers des possibilités abstraites ou, du moins, abstractives. Vers la même époque, Degas réalise un remarquable tableau, Petites paysannes se baignant à la mer vers le soir (1875-76), tableau d'envergure moderne dans sa saisie du mouvement du corps naturel, et sa dissolution des figures qui dansent dans l'eau. Une vision de la liberté féminine - et plus largement humaine - est créée par l'énergie du traitement, par son audace vitale, par son impulsion ${ }^{22}$. En tournant le dos aux canons de beauté féminine, le peintre libère le corps dans sa mouvance et dans son naturel ; par ailleurs, le peintre engage la représentation plus abstractive du corps d'une manière qui met en valeur la joie de vivre dans un style qui anticipe les rondes allègres, profondément humaines, réalisés par Matisse trente ans plus tard dans Le bonheur de vivre (1905-06) et La danse (1910). ${ }^{23}$

Un nouvel humanisme ressort ainsi aux valeurs impressionnistes plus souples de tableaux où l'interrogation critique de la beauté normative passe par la libération du corps et ouvre la voie à la libération de l'esthétique.

\section{Conclusion}

La tradition des ondines mythiques transformées en nymphes réelles des bassins privés et en nus des baignoires et tubs domestiques s'allie à d'autres transformations et innovations picturales, littéraires et esthétiques (illusionnisme, réalisme, impressionnisme, abstractionnisme). Saisir certains de ces déplacements et mutations esthétiques fut l'objectif de cet article.

En guise de conclusion, je rapprocherai encore une fois ces notions de tradition et d'abstraction en proposant un point d'orgue allégorique : le somptueux tableau de Frederick Leighton, Flaming June (1895). ${ }^{24}$ Or, visionner la beauté sensuelle de la femme envoûtée, envoûtante, inspiratrice de rêves d'évasion, c'est lire ce tableau de l'époque victorienne au premier degré. Si le cadre aquatique et les décors classiques de Leighton ont pour fonction apparente d'institutionnaliser la forme féminine somnolente, vouée dans sa volupté à la passivité picturale, en vue de sublimer la beauté canonique, ceci n'est pas tout. Une lecture au deuxième degré s'invite...

\footnotetext{
${ }^{22}$ Tableau accessible via le lien suivant : <https://commons.wikimedia.org/wiki/File:Edgar_Degas_-_Petites_paysannes_se_ lavant_\%C3\%A0_la_mer_vers_le_soir.jpg >. Consulté le : 21 avr. 2021.

${ }^{23}$ Tableaux accessibles via les liens suivants : <https://collection.barnesfoundation.org/objects/7199/Le-Bonheur-de-vivre-also-called-The-Joy-of-Life/> ; <https://www.hermitagemuseum.org/wps/portal/hermitage/digital-collection/01.+Paintings/28411/>. Consultés le : 21 avr. 2021.

${ }^{24}$ Tableau accessible via le lien suivant : <https://artsandculture.google.com/story/flaming-june-by-lord-frederic-leighton/ jAKCa7FDV8CtJA>. Consulté le : 21 avr. 2021.
} 
La femme, porteuse de robe, est porteuse de couleur ; ou plutôt la couleur est ici porteuse de l'idée et de la choséité de la représentation ; le réel de la figure féminine semble se dissoudre dans la saturation orange, semble tourner à l'abstraction. L'oeil du regardant est attiré vers la courbe du corps, vers sa forme incurvée ; son regard est entraîné dans un mouvement quasi-circulaire, ce qui fait que le corps dans son face-à-face traditionnel est défait, décomposé, et l'image résiste au télos narratif pour devenir une allégorie de l'art moderne, synthétisant la forme et la couleur. L'allégorie de l'art créée par Leighton, peintre de réputation arrière-garde, semble rejoindre la perspective de Baudelaire, poète moderne, qui puise dans la double source du passé et du contemporain dans "Le cygne », poème icône où - réel et idéal, matériel et mythique - " tout pour moi devient allégorie » (BAUDELAIRE, T. I, 1975, p. 85-87). ${ }^{25}$ Cette figure picturale des plus poétiques résume - dans sa lucidité percutante, son pouvoir de ravissement, et sa capacité expansive - les tensions entre esthétiques classicisante et innovante, académiste et moderniste, réaliste et irréaliste, et ouvre la voie à de nouvelles synthèses et transfigurations.

\section{RÉFÉRENCES}

BARRINGER, Tim; PRETTEJOHN, Elizabeth (Éds.). Frederic Leighton : Antiquity, Renaissance, Modernity. London : Yale University Press, 1999.

BARROW, Rosemary. The Scent of Roses : Alma-Tadema and the Other Side of Rome. Bulletin of the Institute of Classical Studies, v. 42, s.n., p. 183-202, 1997-98.

BAUDELAIRE, Charles. GEuvres complètes. Ed. Claude Pichois. Paris : Gallimard, 1975. (Bibliothèque de la Pléiade).

BRETELL, Richard. Modern Art : Capitalism and Representation. Oxford : Oxford University Press, 1999.

BROOKS, Peter. Bodywork : Objects of Desire in Modern Narrative. Cambridge : Cambridge University Press, 1993.

BROWN, Kathryn (Ed.). Perspectives on Degas. London : Routledge, 2017.

COLLEY, Ann C. Wild Animal Skins in Victorian Britain : Zoos, Collections, Portraits, and Maps. London : Ashgate, 2014.

CORBETT, David Peters : PERRY, Lara (Éds.). English Art, 1860-1914 : Modern Artists and Identity. Manchester : Manchester University Press, 2000.

CROS, Charles. Le Coffret de santal ; Le Collier de griffes. Paris : Flammarion, 2011.

\footnotetext{
${ }_{25}$ Pour une importante revalorisation de l'œuvre de Leighton, voir l'argument de Elisabeth Prettejohn (in CORBETT et PERRY, 2000, p. 31-48). Selon Prettejohn, l'évacuation de l'histoire et la concentration sur la forme chez Leighton et, propose-t-elle, chez les peintres académiques français, relèvent du progrès réalisé vers un art autonome qui fait abstraction de l'histoire. Voir également Barringer et Prettejohn, qui regroupent des travaux révisionnistes dont certains consacrés à l'étude de la modernité picturale de Leighton. L'essai de Paul Barlow, «Transparent Bodies, Opaque Identities : Personification, Narrative and Portraiture » (in BARRINGER ; PRETTEJOHN, 1999, p. 193-230), propose une analyse de Flaming June, attentive au refus d'un composant narratif et au mode d'abstraction mis en œuvre par Leighton (1999, p. 199-210).
} 
DAWKINS, Heather. The Nude in French Art and Culture. Cambridge : Cambridge University Press, 2002. DUPOUY, Alexandre. Erotic Photography. London : Parkstone, 2003.

FLAUBERT, Gustave. CEuvres complètes. Ed. Claudine Gothot-Mersch et al. Paris : Gallimard, 2013. (Bibliothèque de la Pléiade)

KENDALL, Richard : POLLOCK, Griselda (Eds.). Dealing with Degas : Representations of Women and the Politics of Vision. New York : Universe, 1992.

LATOUR, Nathalie. Céroplasties, corps immortalisés. Paris : Du Murmure, 2020.

KRISTEVA, Julia. Degas. Le Tub. Julia Kristeva, 30 juin 2014. Disponible en : <http://www.kristeva.fr/degas. html>. Consulté le : 21 avr. 2021.

MIRZOEFF, Nicholas. Bodyscape : Art, Modernity and the Ideal Body. London/New York : Routledge, 1995.

PRETTEJOHN, Elizabeth. Lawrence Alma-Tadema and the Modern City of Ancient Rome. The Art Bulletin, v. 84, n. 1, p. 115-129, mars 2002.

RIMBAUD, Arthur. Euvres poétiques. Ed. Antoine Adam. Paris : Gallimard, 1972. (Bibliothèque de la Pléiade).

ZOLA, Émile. Les Rougon-Macquart. T. I. Ed. Henri Mitterand. Paris : Gallimard, 1960. (Bibliothèque de la Pléiade).

ZOLA, Émile. Les Rougon-Macquart. T. II. Ed. Henri Mitterand. Paris: Gallimard, 1961. (Bibliothèque de la Pléiade). 